\title{
Aprendizaje como proceso transformador: una experiencia de proyección a la comunidad
}

Por: M.L. Lucía Alvarado Cantero', Universidad de Costa Rica

Recibido: 11 de marzo, 2017.

Aprobado: $\quad 25$ de mayo, 2017

\section{Resumen}

El docente de español como lengua extranjera o segunda, tiene la oportunidad -y la obligación- de comprometerse con su propio aprendizaje, el de sus estudiantes y con la sociedad en procura de convertir la experiencia pedagógica en un proceso que busque no solo logros a nivel académico, sino transformación personal mediante la exposición a situaciones, textos y personas que permitan sensibilizar a los estudiantes y los impulsen a ser agentes de cambio. La investigación que dio origen a este artículo parte de la noción de docente como intelectual transformador (Dewey, 1977; Freire, 2012 y 2015; Giroux, 1988; Grundy, 1987; Kumaravadivelu, 2003) y de los principios del Aprendizaje Servicio (ApS) y el Aprendizaje Basado en la Comunidad (ABC) (Bringle y Hatcher, 1996; Puig, 2015; Mooney y Edwards, 2001; Rodríguez, 2014; Naval, García, Puig y Santos, 2011; Barreneche y Ramos-Flores, 2013; Ebacher, 2013; Escofet, Folgueiras, Luna y Palou, 2016). El trabajo midió la percepción de 66 estudiantes de ELE en relación con dos variables: mejora de sus habilidades en español y sensibilización hacia poblaciones vulnerables, después de tomar un curso en el que el $20 \%$ de las horas lectivas se dedicó a diseñar y ejecutar proyectos con comunidades. Los resultados obtenidos son satisfactorios y permiten plantear retos para la enseñanza de lenguas y la educación en general.

\section{Abstract}

\section{Learning as A transforming process: An Experience of Community Outreach}

The teachers of Spanish as a mother tongue or a second language has the opportunity -and the responsibility- to commit themselves with their own learning, their students and with society in order to turn the pedagogical experience into a process which not only seeks achievement at the academic level but also the social transformation through the exposure to situations, texts and people that allow them to become more sensitive to students and stimulate them to become agents of change. The research which originated this article departs from the notion of teachers as social transformers (Dewey, 1938; Freire, 2012, 2015; Giroux, 1988; Grundy, 1987; Kumaravadivelu, 2003) and the principles of Social Learning (ApS) and Community-Based Learnin (ABC) (Bringle and Hatcher, 1996; Puig, 2015; Mooney and Edwards, 2001; Rodríguez, 2014; Naval, García, Puig and Santos, 2011; Barreneche and Ramos, 2013; Ebacher, 2013; Escofet, Folgueiras, Luna and Palou, 2016). The paper measured the perception of 66 ELE students in relation to two variables: improvement of their language skills and the sensitivity to vulnerable populations, after taking a class in which $20 \%$ of teaching hours was devoted to the design and execution of community projects. The results that were obtained are satisfactory and allow the setting of challenges for the teaching of languages and education in general.

1 Lucía Alvarado Cantero es bachiller en Filología Española y Máster en Español como Segunda Lengua por la Universidad de Costa Rica. Colaboradora en la Academia Norteamericana de la Lengua y se desempeña como docente de las Escuelas de Filología, Lingüística y Literatura y de Ciencias de la Comunicación Colectiva, en la Universidad de Costa Rica. Contacto: alvarado.lucia@gmail.com.

\footnotetext{
PALABRAS CLAVE:

Aprendizaje Servicio, Aprendizaje Basado en la Comunidad, conversación con proyección a la comunidad, docente como intelectual transformador, educación como agente de cambio, español como lengua extranjera, español como segunda lengua.

\section{KEY WORDS:}

Service Learning, Community-based Learning. Conversation and contact with the community, teachers as transforming Educators, education as an agent of social change, Spanish as a foreign language, Spanish as a second language.
} 
¿Por qué no discutir con los alumnos la realidad concreta, a la que hay que asociar la materia cuyo contenido se enseña: la realidad agresiva, en que la violencia es una constante y la convivencia de las personas con la muerte es mucho mayor que con la vida? ¿Por qué no establecer una "intimidad" necesaria entre los saberes curriculares fundamentales para los alumnos y la experiencia social que ellos tienen como individuos? ¿Por qué no discutir las implicaciones políticas e ideológicas de la falta de atención de los dominantes en relación con áreas pobres de la ciudad? ¿Y la ética de clase incrustada en esa desatención? Porque, dirá un educador reaccionariamente pragmático, la escuela no tiene nada que ver con eso. La escuela no es partido. Ella tiene que enseñar los contenidos, transferirlos a los alumnos. Una vez aprendidos, estos operan por sí mismos (Freire, 2012, p. 32).

\section{INTRODUCCIÓN}

La enseñanza de lenguas -y la educación en general- ha experimentado dos cambios significativos en el último medio siglo: por una parte, una sociedad industrial basada en la producción masiva de objetos, servicios e información; por otra, los descubrimientos científicos nos han permitido conocer mejor cómo funciona el cerebro, órgano encargado del aprendizaje (Punset, 2011). Este conocimiento nos confirma algo que los griegos ya sabían: que se aprende, no repitiendo, sino haciendo, cuando nos emocionamos (íd.).

Los voluntariados, los programas de inmersión y los intercambios entre países han buscado integrar la práctica -a la que se refiere Punset (2011) - y la interacción con hablantes nativos como medio para mejorar las competencias comunicativas en la lengua meta. Estos programas se han fortalecido con el requisito de hacer estudios en el extranjero o de participar en proyectos con la comunidad durante por lo menos un semestre en algunas universidades europeas (por medio de diversos programas, entre los que destaco Erasmus ${ }^{1}$, por ejemplo) y estadounidenses (Pellettieri, 2011). En Costa Rica, es común que en los centros de enseñanza de español para extranjeros se incluyan giras a comunidades y trabajos comunitarios, entre otros.

A partir de los años noventa, se comenzaron a sistematizar estas actividades de interacción con hablantes nativos -para el caso del español, tanto para entornos de inmersión como en los países de origen que cuentan con población hispanohablante- a partir de metodologías como el Aprendizaje Basado en la Comunidad y el Aprendizaje Servicio (Naval, García, Puig y Santos, 2011). Estas iniciativas surgieron con fuerza en los Estados Unidos y en Argentina (Sotelino, 2014) y con el tiempo se han ido integrando a los programas de lengua de universidades e institutos en España y Latinoamérica.

Esta investigación presenta los resultados de la implementación de un programa diseñado a partir del ApS, en el curso Conversación con proyección a la comunidad I, II, III, el cual formó parte de la oferta académica del Programa de Español como Lengua Extranjera de la Universidad de Costa Rica en el periodo enero-noviembre, 2016. En las siguientes páginas, se describen los fundamentos teóricos y metodológicos que fundamentan el programa del curso y se analizan las evaluaciones por parte de 66 estudiantes, quienes tomaron el curso en diferentes momentos en el periodo mencionado. Las evaluaciones se aplicaron por medio de encuestas que buscaban medir la percepción de los estudiantes en relación con el mejoramiento de sus habilidades lingüísticas y con su proceso de sensibilización con la comunidad, luego de tomar el curso.

La innovación de este trabajo es tal que en Costa Rica puesto que, hasta ahora, no se ha medido en ninguna casa de enseñanza de español como segunda lengua o lengua extranjera en el país el impacto que las actividades con la comunidad tienen en la percepción de los estudiantes respecto de sus procesos de aprendizaje y sensibilización.

\section{EXPERIENCIAS PREVIAS DE APS Y ABC}

Ebacher (2013) organizó en su universidad en Maryland un programa de ApS en el cual los estudiantes

1 Para más información, puede consultarse su página: http://oapee.es/oapee/inicio/pap/erasmus.html. 
de español participaban como intérpretes en la oficina del alcalde en una clínica de planificación familiar. Los resultados de este programa mostraron que los objetivos lingüísticos no solo se alcanzaron, sino que se sobrepasaron; las actividades de traducción y de interpretación, por su parte, proveyeron a los estudiantes de experiencias reales de uso de la lengua que fueron valoradas como positivas. Esta autora, retoma a Hale (1999, citado por Ebacher, 2013) y relaciona el éxito de su programa con la idea de que la epistemología del aprendizaje-servicio se basa en la asunción de que el conocimiento se obtiene mediante procesos interactivos de acción y reflexión. El aprendizaje, en aprendizaje-servicio, ocurre mediante la experiencia. Gracias al aprendizaje-servicio en la comunidad, los estudiantes toman parte activa en la creación del conocimiento. Esta creación es el resultado de un proceso de interacción y reflexión por parte del estudiante.

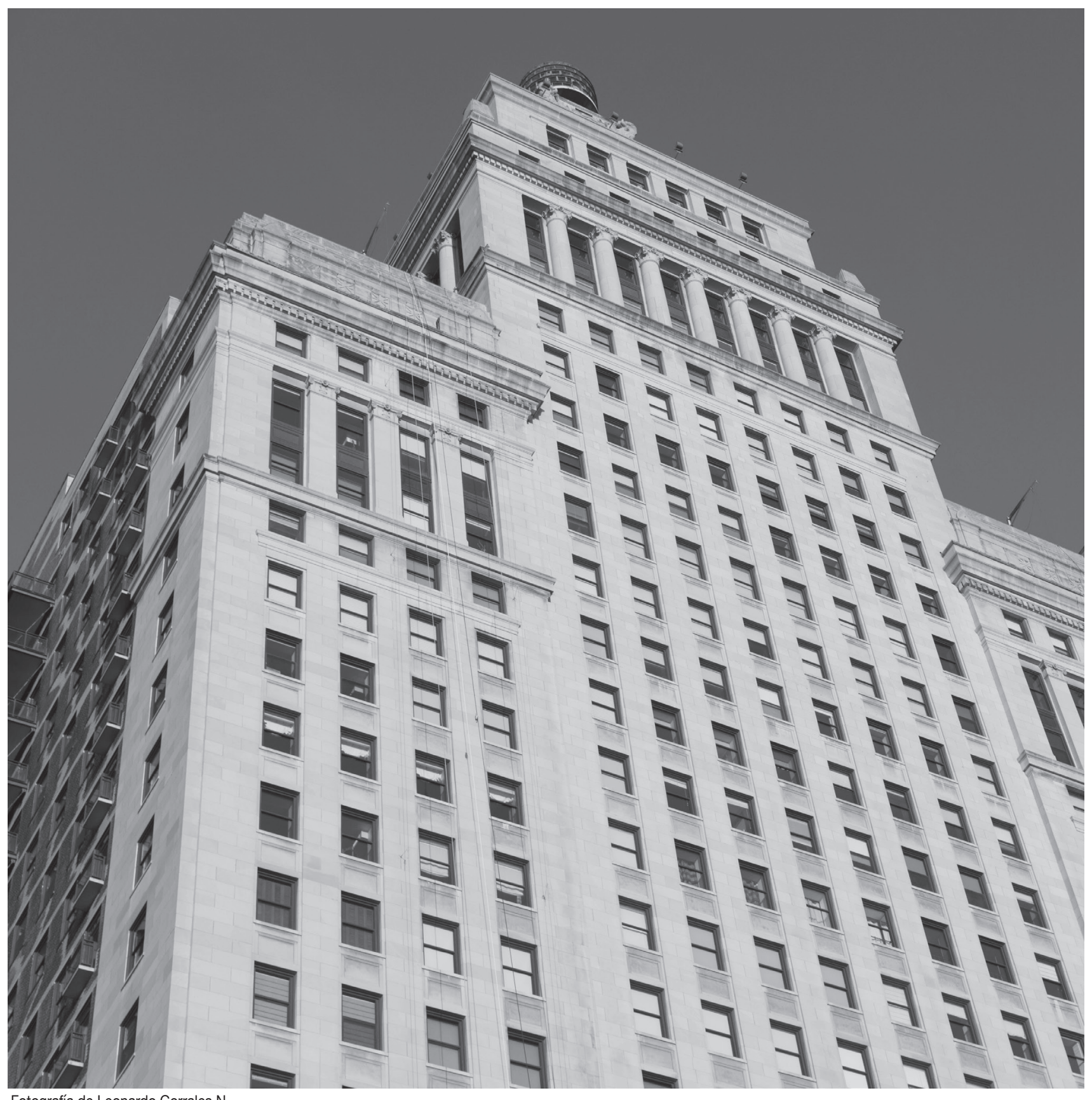

Fotografía de Leonardo Corrales $\mathrm{N}$. 
Los resultados obtenidos por Ebacher (2013) se replican en otras investigaciones. Llorente (2003) concluyó, luego de aplicar un programa de ApS, que el aprendizaje a través del servicio ayuda a reducir los estereotipos y contribuye a la comprensión cultural y racial. No se puede dejar de lado que el aprendizaje académico tiende a recibir una influencia positiva por la aplicación de la teoría aprendida en la clase. Esta última afirmación es compartida por Tacelosky (2013), cuya investigación reveló que, al finalizar el proyecto de ABC, los estudiantes afirmaron haber podido relacionar los contenidos vistos en clase con contextos reales. De esta manera, el ApS y el ABC no solo proveen un servicio a la comunidad, sino que ayudan a los estudiantes a cumplir con los objetivos de aprendizaje del curso, ya que mejora las competencias lingüística y cultural (Faszer-Mcmahon, 2013) e incide en una evaluación positiva de sus destrezas de habla (Pellettieri, 2011).

El Aprendizaje Basado en la Comunidad (ABC) se refiere a cualquier herramienta pedagógica en la cual la comunidad se convierte en parte del proceso de aprendizaje. Todas las iniciativas de ABC son experienciales. Desde esta perspectiva, el ApS es una instancia del ABC (Mooney y Edwards, 2001).

La metodología ApS les permite a los estudiantes un mayor dominio del contenido de la asignatura, despierta su capacidad de asumir responsabilidades serias, aumenta la autoestima y la autosuficiencia, incrementa habilidades de trabajo en equipo, fomenta su deseo de superación, mejora la comunicación y despierta la creatividad en los alumnos (Rodríguez, 2014). Desde la perspectiva del ApS, la institución educativa no solo se convierte en un espacio de diálogo, sino que entra en diálogo con la comunidad y conforma una relación recíproca, con intereses y corresponsabilidades definidas (Escofet, Folgueiras, Luna y Palou, 2016).

A nivel personal, los estudiantes que se integran en programas de ApS y $A B C$ perciben que su eficacia personal y sus habilidades comunicativas mejoran, experimentan un crecimiento personal, paralelo al académico y social (Llorente, 2003; Tacelosky, 2013); asimismo, estos programas aumentan la motivación en su proceso de aprendizaje de la lengua (Faszer-McMahon, 2013; Pellettieri, 2011; Rodrí- guez, 2014) y el deseo de integrarse a la comunidad y los impulsan a conectar con hablantes de español fuera de la clase (Faszer-McMahon, 2013). Se ha evidenciado, adicionalmente, el fortalecimiento de la confianza al hablar español y más disposición para hacerlo (Pellettieri, 2011; Zapata, 2011), lo cual favorece, consecuentemente, la prosociabilidad (Rodríguez, 2014). Por otra parte, las actitudes de los estudiantes hacia la lengua y hacia la cultura meta se modifican positivamente posterior a una experiencia de ApS (Rodríguez, 2014; Zapata, 2011).

La interacción con la lengua auténtica contribuye para que los estudiantes identifiquen variedad de registros de uso en el habla y de diferencias de uso de la lengua en diferentes entornos educativos y socioeconómicos; genera discusión y reflexión informadas sobre asuntos socioculturales y socioeconómicos relacionados con los problemas y las situaciones de desigualdad y exclusión de las comunidades (Faszer-McMahon, 2013). Estos procesos propician una reevaluación de los sistemas de creencias de los discentes a la luz de lo que viven en los nuevos contextos de enseñanza que ponen a su disposición las actividades de ApS y ABC (Jovanovic y Filipovic, 2013).

En cuanto al uso de este tipo de programas por parte de los docentes, Hellebrant y Jorge (2013) obtuvieron resultados interesantes en una encuesta a 805 docentes de español en los Estados Unidos: la mayoría de las personas encuestadas está interesada en integrar este tipo de programas en sus cursos; sin embargo, de esta muestra, solamente un grupo pequeño realmente lo logra. En cuanto a los tipos de Participación en la Comunidad (CE por sus siglas en inglés) que emplean, el voluntariado fue el referido con más frecuencia, seguido por Aprendizaje Servicio, Aprendizaje Basado en la Comunidad y, con menor frecuencia, se mencionaron los internados y las investigaciones basadas en la comunidad. Sotelino (2014) halló resultados similares en una investigación de corte parecido en Galicia: los profesores (326, en total) valoraron de manera muy positiva la participación de sus alumnos en las sesiones de aula; no obstante, encuentra que el profesorado desconoce en su gran mayoría el aprendizaje-servicio y no ha realizado actividades de ese tipo, aunque sí 
consideran positiva la posibilidad de incorporar esta metodología a las aulas.

Como parte de los obstáculos en la implementación de estos programas se destacan las barreras institucionales: falta de recursos, poco apoyo económico y político (Hellebrant y Jorge, 2013) y el hecho de que su implementación requiere trabajo adicional (Rodríguez, 2014).

\section{ENFOQUE TEÓRICO}

Para rastrear las bases de ApS es necesario recurrir a los autores pioneros en señalar la relación intrínseca entre la educación, el contexto social y la comunidad. Todo esto en un contexto histórico en el que, además de fundamentar sólidamente una crítica a los sistemas de enseñanza tradicionales basados en la transmisión de conocimientos, se atrevieron a proponer maneras alternativas de concebir la educación, los roles docente y discente y la escuela. Por una parte, Freire (2015) a partir de su pedagogía liberadora entiende la educación como acercamiento crítico a la realidad, este acercamiento está, a su vez, mediado por la noción de concienciación:

La concientización no es estar frente a la realidad asumiendo una posición falsamente intelectual, por lo tanto intelectualista; (...) la concientización no puede existir fuera de la praxis, esto es, fuera de la acciónreflexión. (...) [La concientización] implica que los hombres asuman el rol de sujetos hacedores del mundo: rehacedores del mundo. La concientización es compromiso histórico, y compromiso histórico exige la transformación al mundo y no la adaptación a él (p. 113).

La educación como instrumento de liberación, entonces, propicia el desarrollo de estos procesos de concientización. El papel del educador se centra en problematizar la realidad concreta del educando, mientras, al mismo tiempo, se educa. Desde esta concepción, se realiza una educación "iluminada por una visión humanista de carácter científico" (Freire, 2015, p. 128), que rechaza la tradición de enseñar para trasmitir contenidos y privilegia la noción de enseñar a "pensar correctamente" (Freire, 2012, p. 28) desde el respeto a los conocimientos previos, a los saberes de los estudiantes y desde procesos docentes de investigación: "no hay docencia sin discencia" (Ibid, p. 28). El fin último de la educación es, de esta forma, que los estudiantes se transformen en "sujetos reales de la construcción y reconstrucción del saber enseñado, al lado del educador, también sujeto del proceso"(Ibid, p. 28).

El segundo autor, John Dewey (1977), enfatiza en la necesidad de que la persona sea educada como individuo social, ya que la educación, según él, es responsable de regular el proceso de participación de los individuos en la conciencia social. Dewey (1977) es tajante al señalar que gran parte de los proyectos educativos fracasa porque olvida el principio fundamental de la escuela como una forma de vida en comunidad. Considera que la educación debe elaborar una reconstrucción continua de la experiencia, y que el proceso y el objetivo de la educación no pueden ser disociados. Este último planteamiento es coherente con la corriente de pensamiento constructivista, para la cual, el sujeto es un constructor activo de sus estructuras de conocimiento, el cual es producto de un proceso y es observable en tanto se desarrolla, no solo en el producto final (Rosas, 2008).

A finales de la década de 1980, Giroux (1988), fuertemente influenciado por las ideas de Freire y Dewey, observa que cualquier intento por reformular el rol de los educadores, debe empezar por cuestionar el propósito de la educación. Al respecto, propone la visión de la escuela como esferas públicas democráticas, sitios dedicados a las formas de alcanzar el ser y el empoderamiento social; estos se construyen desde la investigación crítica que dignifica el diálogo significativo y la agentividad humana. Los estudiantes aprenden, de esta forma, un discurso asociado a la asociación pública y a la responsabilidad social, este discurso busca fusionar la libertad individual y la justicia social con el fin de formar seres humanos críticos, con un alto sentido cívico.

Uno de los aportes más sobresalientes de este autor es su contribución en la discusión sobre el rol del docente. Al respecto, es contundente al afirmar que la tarea primordial del educador es ser un intelectual transformador que desarrolle pedagogías contrahegemónicas, las cuales deben buscar no solo empoderar a los estudiantes a partir del conocimiento sino 


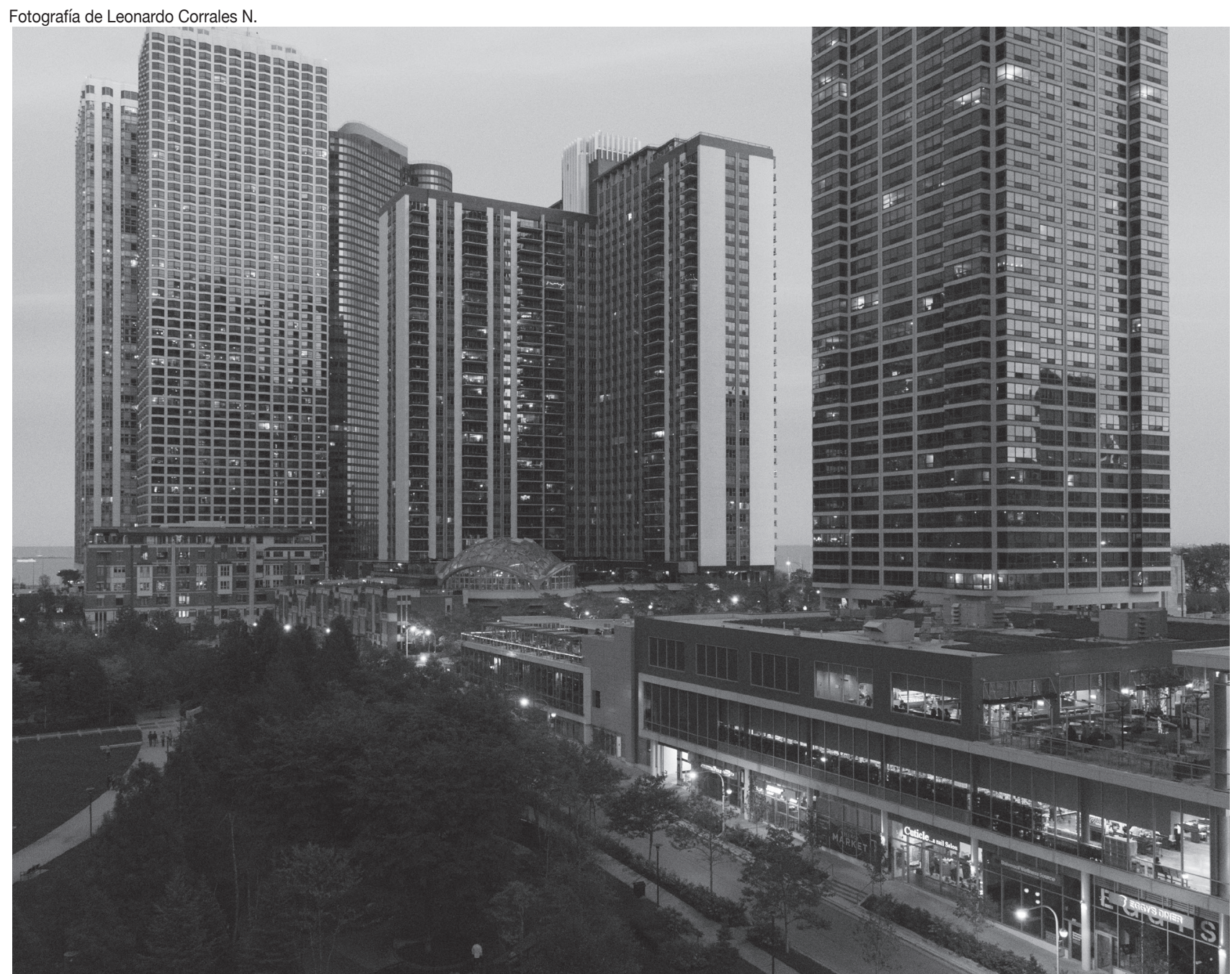

también desde las habilidades sociales que necesitan para ser agentes críticos en la sociedad:

As intellectuals, they [the teachers] will combine reflection and action in the interest of empowering students with the skills and knowledge needed to address injustices and to be critical actors committed to developing a world free of oppression and exploitation. (...) They are concerned with empowering students so they can read the world critically and change it when necessary (Giroux, 1988, p. 319).

En una línea de pensamiento similar, Grundy (1987) se refiere a la organización de los programas para la enseñanza de lenguas. Parte de la idea de currículum no como concepto sino como construcción cultural, una forma de organizar un conjunto de prácticas educativas humanas. Para esta autora, toda práctica educativa supone una forma de concebir el ser humano en el mundo. Por esto, las prácticas educativas, y el currículo como parte de estas, no existen separadas de las creencias sobre las personas y sobre la forma en que interactúan y deben hacerlo en el mundo. Entonces, si trascendemos los aspectos superficiales de la práctica educativa -lo que suponen las prácticas de organización educativa así como sus implicaciones y lo que suponen las prácticas de organización y de enseñanza y aprendizaje- no hallamos leyes naturales universales, sino creencias y valores (Grundy, 1987, p. 22).

Grundy (1987) fundamenta su propuesta en la noción de "interés constitutivo del conocimiento" como una orientación de la especie humana hacia el conocimiento o la racionalidad. Este interés configura lo que constituye el conocimiento y determina las categorías mediante las cuales es organizado. 
Basada en Habermas (1974), propone tres tipos de intereses, los cuales conforman tres tipos de ciencia mediante los que se genera y organiza el saber en nuestra sociedad:

a. Técnico: interés fundamental por el control del ambiente mediante la acción, de acuerdo con reglas basadas en leyes con fundamento empírico. Está implícito el interés por el control del aprendizaje del alumno a partir de objetivos que el estudiante deberá cumplir al finalizar el proceso de enseñanza.

b. Práctico: interés fundamental por comprender el ambiente mediante la interacción, basado en una interpretación consensuada del significado. El currículum informado por el interés práctico no es del tipo medios-fin, en el que el resultado educativo se produce mediante la acción del profesor sobre un grupo de alumnos objetivados. En cambio, el diseño del currículo se considera como un proceso en el que alumno y profesor interactúan con el fin de dar sentido al mundo.

c. Emancipador: da lugar a la acción autónoma, responsable, basada en prudentes decisiones informadas por cierto tipo de saber. Mientras los otros dos intereses se ocupan del control y de la comprensión, el emancipador se preocupa de la potenciación, o sea, de la capacitación de individuos y grupos para tomar las riendas de sus propias vidas de manera autónoma y responsable.

Desde el enfoque curricular emancipatorio, se privilegian las experiencias de aprendizaje significativas, entendiendo estas como aquellas en las cuales se evidencia la praxis, no como la realización de una acción sobre la cual se ha reflexionado críticamente, sino como la que involucra una escogencia libre para actuar, que ha sido orientada por reconstrucciones del mundo social. Desde esta perspectiva: la praxis no es una acción que mantiene una situación tal como es en el presente, sino una acción que cambia tanto el mundo como la propia comprensión de él (Agray, 2010, p. 421).

Kumaravadivelu (2003), más recientemente, relaciona la categorización de intereses propuesta por Grundy (1987) con los roles asociados a la práctica docente en el contexto de la enseñanza de lenguas y define tres perfiles: el profesor como técnico pasivo, el profesor como practicante reflexivo y el docente como intelectual transformador.

El primero, el técnico pasivo, se define desde la escuela conductista, la cual enfatiza la importancia de la verificación científica. Desde esta perspectiva, el enfoque principal de la enseñanza está en el profesor y en los contenidos, los cuales consisten en hechos verificados y verificables y en reglas claramente articuladas. Este corresponde, en términos de Freire (2012), al intelectual memorizador: aquel que se domestica ante el texto, con miedo de arriesgarse, habla de sus lecturas casi como si estuviera recitando de memoria, no percibe ninguna relación, cuando realmente existe, entre lo que leyó y lo que ocurre en su país, en su ciudad, en su barrio (p. 28). El rol principal de este docente técnico es funcionar como un conducto, canalizar el flujo de información desde un punto del espectro educativo (el experto) al otro (el estudiante) sin alterar significativamente el contenido (Kumaravadivelu, 2003); en otras palabras, transmitir información (Freire, 2012).

El docente como practicante reflexivo, sigue la noción de enseñanza de Dewey (1977), según la cual, esta no está conformada por una secuencia de procesos sino por acciones sensibles al contexto enraizadas en un pensamiento intelectual. Los docentes son vistos no como transmisores pasivos sino como agentes capaces de solucionar problemas. La enseñanza reflexiva es, por lo tanto, un enfoque holístico que enfatiza la creatividad, el arte y la sensibilidad al contexto. Por lo general, los roles de este docente son examinar y tratar de resolver problemas en clase; estar al tanto de preguntas, asunciones y valores que pueda traer a la enseñanza; estar atento a los contextos institucionales y culturales en los cuales enseña; tomar parte en el desarrollo del curriculum e involucrarse en los esfuerzos de cambio en la escuela y responsabilizarse por su desarrollo personal (Kumaravadivelu, 2003).

A pesar de la apertura que implica pasar de un modelo de técnico pasivo a uno de practicante reflexivo, Kumaravadivelu (2003) señala tres carencias que comporta este tipo de formación docente: en primer lugar, se enfoca solamente en el rol del profesor, por 
lo que tiende a tratar la reflexión como un proceso introspectivo; en segundo lugar, al concentrarse en las acciones del docente en el aula, no presta suficiente atención a los factores sociopolíticos que forman y conforman la práctica docente; finalmente, no logra desvincularse de la creencia de que los contenidos válidos para llevar al aula son, precisamente, los que provienen de fuentes académicas validadas por instituciones.

Kumaravadelu (2003) culmina su propuesta tríadica de roles docentes con el intelectual transformador, definición que evidencia fuertes influencias de los trabajos en pedagogía crítica de Freire y Giroux, en cuanto a que parte de una visión del docente como un profesional que debe reflejar los principios ideológicos que nutren su práctica, conectar la teoría pedagógica y la práctica de asuntos sociales más amplios, para, como fin último, reflejar en su ejercicio docente una visión de una vida mejor y más humana.

Como transformadores intelectuales, continúa el autor, los docentes tienen una tarea dual, deben preocuparse por el avance educativo de sus estudiantes, pero también por su transformación personal. Para alcanzar el avance educativo, el profesor debe comprometerse con la creación e implementación de formas de conocimiento que sean relevantes para los contextos específicos de enseñanza, así como con la construcción de programas y cursos que se basen en las necesidades, deseos y situaciones particulares de los discentes. Este imperativo exige de los maestros una potenciación de la conciencia sociopolítica en el grupo por medio del uso de actividades cuyos objetivos giren en torno a la resolución de problemas y a la concienciación (Kumaravadivelu, 2003).

Para lograr la transformación personal, los docentes deben educarse por su propia cuenta, al tiempo que se esfuerzan por educar a sus estudiantes en cuanto a las formas en que se expresan, dirigen y redirigen la desigualdad y la injusticia en la sociedad (íd.). El rol dual del docente, por lo tanto, "requires teachers to view pedagogy not merely as a mechanism for maximazing learning opportunities in the classroom but also as a means for transforming life in and outside the classroom" (Ibid, p. 14).
Kumaravadivelu (2003), basado en Kincheloe (1993), resume las características del intelectual transformador de la siguiente manera: orientado a la investigación, contextualizado socialmente, comprometido con acciones para mejorar la interacción profesor-estudiante, dedicado al arte de la improvisación y a cultivar participaciones situadas, reflexivo en asuntos sociales y con capacidad para la autocrítica, comprometido con la educación libre y autodirigida, sensible a la diversidad cultural y lingüística de los discentes, comprometido con la acción en la docencia y preocupado por la dimensión afectiva de los seres humanos.

Las ideas de Dewey (1977), Freire $(2015,2012)$ y Giroux (1988) han delineado una orientación específica en la concepción de las acciones relativas a la educación. La enseñanza de lenguas no ha estado exenta de esta influencia. En este sentido, el ApS surge como un método cuyo objetivo es integrar actividades de inmersión con la comunidad en los cursos académicos con el fin de mejorar los objetivos lingüísticos específicos al curso y de funcionar como un vehículo efectivo para enseñar a los estudiantes sobre compromiso cívico (Barreneche y Ramos, 2013). El ApS pone en relación el aprendizaje académico con algún proyecto de colaboración con la comunidad, en este binomio "aprendizaje académico-trabajo en la comunidad", como espacio que se enriquece desde ambas partes, es donde reside la sustantividad del aprendizaje-servicio (Escofet, Folgueiras, Luna y Palou, 2016).

Ahora bien, la definición más extendida de ApS ha sido tomada de Bringle y Hatcher (1996):

Método de aprendizaje en el que los estudiantes ofrecen servicios a sus comunidades a cambio de créditos académicos, usando y ejercitando destrezas con tiempo para reflexionar sobre la actividad de aprendizaje servicio de forma que obtengan mayor comprensión del contenido del curso, una más amplia apreciación de la disciplina y sentido de la responsabilidad cívica intensificado (p. 112).

En esta definición, el ApS se ve como un método, sin embargo, otros autores lo han considerado una propuesta de estrategia metodológica, una metodología o incluso una propuesta pedagógica (Escofet et al., 
2016). Puig (2015), por su parte, plantea el ApS como un programa en el que deben estar presentes once elementos fundantes: compromiso cívico; articulación de tres dinamismos educativos básicos: necesidades, servicio y aprendizaje; experiencia significativa que incluya participación, cooperación, reflexión y reconocimiento; relaciones de colaboración entre los centros educativos y las entidades sociales; compromiso personal e inteligencia pedagógica en la incorporación del aprendizaje servicio a un centro educativo; reconocimiento de su dimensión pedagógica y definición de un servicio útil y formativo; seguimiento de etapas bien establecidas y abiertas a cambios imprevistos; enfoque en las competencias en la evaluación; aplicación a múltiples ámbitos temáticos; estrategias locales en el desarrollo de los proyectos y análisis del proyecto con la ayuda de rúbricas.

Así, mediante los proyectos de aprendizaje-servicio la institución educativa se transforma en un espacio interno de participación, de creación de democracia y desarrollo de visión común que busca una línea de actuación por aprender y actuar en conjunto; en un espacio que aúna y articula esfuerzos con otras instituciones y entidades de la comunidad, que integra objetivos curriculares y necesidades comunitarias y que plantea un trabajo coordinado que fortalece el tejido social y contribuye a la creación de capital social; y en un promotor de la necesaria corresponsabilidad entre los distintos agentes educativos, formales y no formales, para dar una respuesta eficaz a los problemas educativos que la actual sociedad plantea (Escofet, Folgueiras, Luna y Palou, 2016).

Las actividades que se desarrollan en el marco de un proyecto de ApS deben seguir tres criterios: en primer lugar, debe ser un servicio relevante y significativo para la comunidad; en segundo lugar, debe mejorar el aprendizaje académico y en tercero, debe propiciar el aprendizaje cívico (Howard, 2001, en Ebacher, 2013). Estas actividades también deben ofrecer al estudiante la posibilidad de entrar en contacto con experiencias auténticas de la lengua, de interactuar con hablantes nativos (Tacelosky, 2013).

En relación con el compromiso cívico, Naval, García, Puig y Santos (2011) concuerdan con las inves- tigaciones Ilevadas a cabo por la IEA (Association for the Evaluation of Educational Achievement), las cuales concluyeron que la integración de la educación cívica en el currículum, incide positivamente en la educación ciudadana y en la promoción de un compromiso cívico y político. Así:

Las instituciones de educación superior tienen la capacidad y la responsabilidad de informar los procesos políticos, y de utilizar los recursos con los que cuenta para la resolución de problemas contemporáneos. Más en concreto, las universidades tienen una responsabilidad cívica de ser agentes de cambio social (Naval et al., 2011, p. 82).

La Universidad de Stanford contribuyó en la delimitación de los factores que se deben integrar en un programa de ApS. En 1996, definió los Cuadrantes del Aprendizaje Servicio, cuyo fin es guiar las actividades que se inscriben en un programa de este tipo y regular la calidad de los objetivos. El ApS, de acuerdo con este marco, debe estar basado en un objetivo lingüístico específico y en una actividad sustantiva con la comunidad que permita la interacción con nativos, asimismo, debe generar un impacto positivo para esa población (Naval et al., 2011).

Una discusión importante que han generado las propuestas de ApS está relacionada con el voluntariado y la diferencia entre ambas. Al respecto, Mooney y Edwards (2001) clasifican ambos eventos como parte del Aprendizaje Basado en la Comunidad (ABC), el cual, como se definió en el apartado anterior, es una herramienta pedagógica en la cual la comunidad se convierte en parte del proceso de aprendizaje. Ahora bien, Rodríguez (2014), establece diferencias claras entre el ApS y el voluntariado, las cuales se ilustran en la siguiente tabla: 
Imagen 1: Diferencias entre el ApS y el voluntariado comunitario

\begin{tabular}{|l|l|}
\hline \multicolumn{1}{|c|}{ APRENDIZAJE SERVICIO } & \multicolumn{1}{c|}{$\begin{array}{c}\text { VOLUNTARIADO Y SERVICIO } \\
\text { COMUNITARIO }\end{array}$} \\
\hline $\begin{array}{l}\text { Enfoque pedagógico-solidario y metodología de } \\
\text { enseñanza-aprendizaje }\end{array}$ & Enfoque pedagógico-solidario \\
\hline Objetivos de servicio y objetivos de aprendizaje & Objetivos de servicio \\
\hline Formación profesional y formación ciudadana & Formación ciudadana \\
\hline $\begin{array}{l}\text { Requiere fases de preparación, acción y } \\
\text { reflexión }\end{array}$ & Comprende la actividad misma \\
\hline $\begin{array}{l}\text { Existen normas establecidas para el desempeño } \\
\text { del servicio }\end{array}$ & No existen normas establecidas \\
\hline $\begin{array}{l}\text { Requiere planteamiento anticipado a la fecha } \\
\text { del servicio }\end{array}$ & Puede ocurrir en cualquier momento \\
\hline $\begin{array}{l}\text { Debe realizarse con la supervisión adecuada de } \\
\text { un adulto }\end{array}$ & Puede realizarse sin supervisión de un adulto \\
\hline Certificado de acreditación del servicio & No es un requisito \\
\hline
\end{tabular}

Fuente: Rodríguez, 2014.

A nivel general, el ApS requiere un nivel de planeación académica mayor, puesto que implica la integración de la materia del curso en la actividad con la comunidad. De aquí que los fines y el tipo de formación del ApS sean más amplios que los del voluntariado. Las últimas tres diferencias propuestas por Rodríguez (2014) son ampliamente discutibles, puesto que, muchos programas de voluntariado son muy rigurosos en su planeamiento, en las fechas en las que se puede llevar a cabo y en la certificación que ofrecen. Además, con frecuencia, las iniciativas de ApS deben integrarse a programas de voluntariado vigentes en el momento de su ejecución.

Barreneche y Ramos-Flores (2013) agregan que una de las diferencias más claras entre el ApS y el voluntariado es el enlace intencional del primero con un reforzamiento de los objetivos del aprendizaje académico de un curso en particular.

En resumen, las actividades de ApS implican algún tipo de voluntariado, sin embargo, este debe ser complementado con un componente lingüístico o académico que se desea mejorar y con una sistematización y estructuración de las actividades que se efectúan con la comunidad. Estas actividades son planeadas por los docentes y por los estudiantes de acuerdo con las necesidades definidas para la comunidad con base en las necesidades comunicativas y personales de los actores involucrados y el impacto proyectado en la sociedad.

Las ventajas a nivel personal, académico y social del ApS para los estudiantes, los docentes y las instituciones de enseñanza han sido comentadas en el apartado anterior y han sido fundamentadas en experiencias docentes previas desarrolladas con éxito en Estados Unidos, Europa e Hispanoamérica. Los resultados de estas investigaciones, así como la teoría expuesta en este segmento, fundamentaron la elaboración de un curso para el Programa de Español como Lengua Extranjera de la Universidad de Costa Rica explicado en el apartado siguiente.

\section{DISEÑO DEL CURSO CONVERSACIÓN CON PROYECCIÓN A LA COMUNIDAD I, II Y III}

La fundamentación teórica de este curso se enriqueció con los planteamientos de Freire $(2012,2015)$, Dewey (1977), Giroux (1988), Grundy (1987) y Kumaravadivelu (2003). El componente metodológico se basa en los principios del Aprendizaje Servicio (Bringle y Hatcher, 1996; Puig, 2015; Mooney y Edwards, 2001; Rodríguez, 2014; Naval, García, Puig 


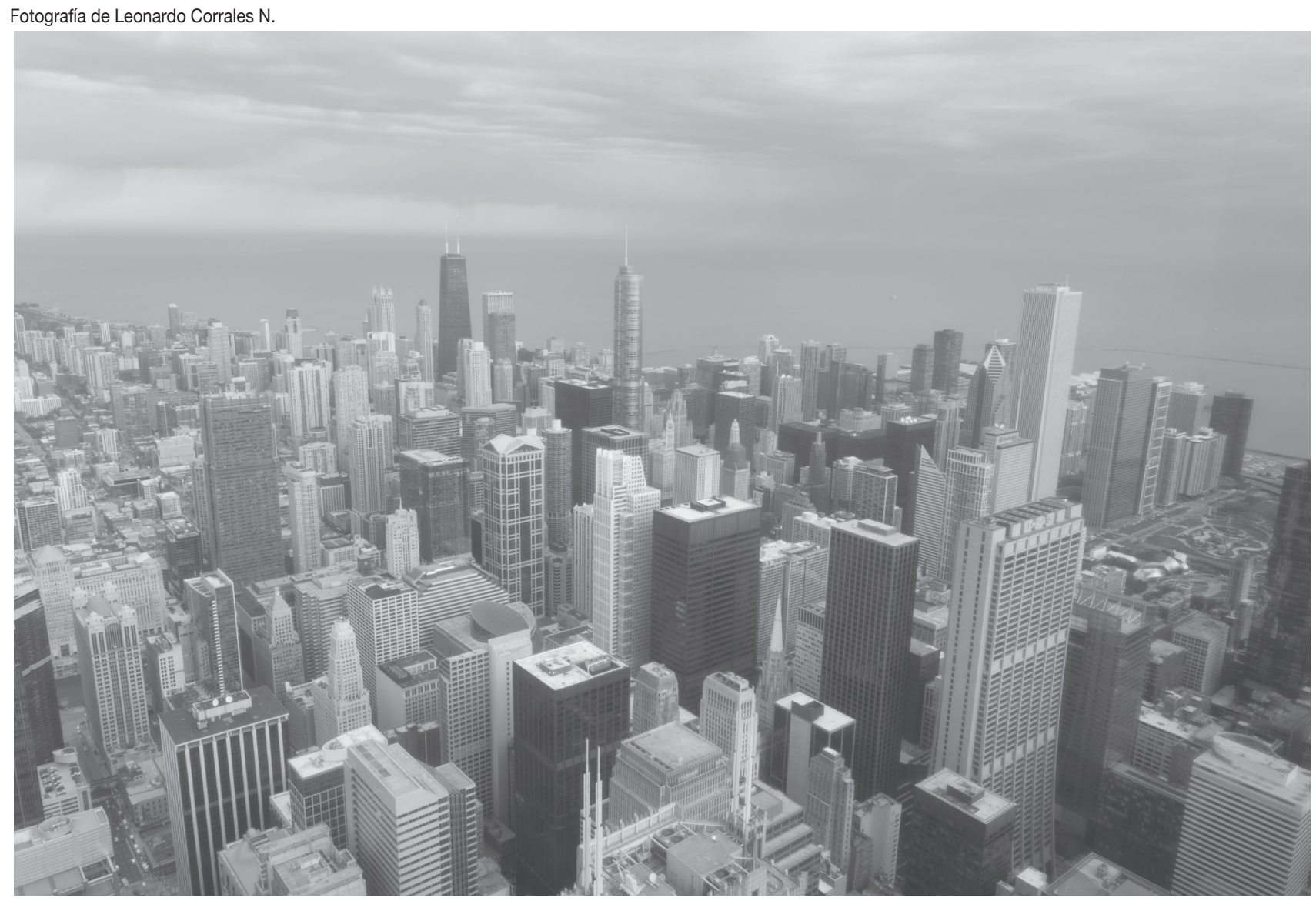

y Santos, 2011; Barreneche y Ramos, 2013; Ebacher, 2013; Escofet, Folgueiras, Luna y Palou, 2016).

El objetivo del curso es orientar al estudiante en la comprensión y uso de estrategias discursivas de interacción oral en español, en particular en el contexto costarricense, tanto en situaciones formales como informales. Se definió una intensidad de 45 horas, cantidad de horas asignadas a todos los cursos del Programa de Español como Lengua Extranjera de la Universidad de Costa Rica. El curso tiene una duración de cuatro semanas y se imparte cada mes. Dado que el curso se enfoca en el mejoramiento de las habilidades de interacción en contextos orales, las horas lectivas se distribuyeron de la siguiente manera:

a. Actividades en clase guiadas por el docente (34 horas): se revisan temas de conversación y estrategias para interactuar en contextos orales formales e informales como conversaciones, exposiciones, entrevistas, entre otros.

b. Proyección a la comunidad (8 horas): trabajo de voluntariado en una institución de ayuda social.
Este trabajo tiene dos objetivos principales: impactar de forma positiva a la comunidad donde se realiza la labor de voluntariado y que el estudiante interactúe con grupos etarios y sociales distintos.

c. Intercambio con hablantes nativos (3 horas): los estudiantes tienen la oportunidad de conversar con otros estudiantes costarricenses. El tiempo de interacción se divide en partes iguales entre la lengua materna de ambos estudiantes.

Los contenidos del curso se sugieren en el programa y están adaptados al nivel de los estudiantes. Para esto, se prepararon tres programas distintos: CO210, dirigido a estudiantes de niveles básicos (A1 y A2 en el Marco Común Europeo de Referencia [MCER]), CO-310, dirigido a estudiantes de niveles intermedios (B1 y B2.1 del MCER) y CO-410, para los niveles avanzados (B2.2 en adelante del MCER); no obstante, estos temas están sujetos a cambios de acuerdo con las necesidades comunicativas, las preferencias y las situaciones de los estudiantes. 
Como parte de la metodología del curso se privilegian las actividades de interacción oral, aunque también se ejercitan las otras destrezas: lectura, escritura y escucha. El curso pretende proveer al estudiante de las herramientas necesarias para mantener conversaciones y para comentar textos especializados cuyo nivel de dificultad variará de acuerdo con el nivel. A nivel general, todos los cursos del Programa de Español como Lengua Extranjera de la Universidad de Costa Rica consideran el aprendizaje como un proceso integral que debe tomar en cuenta las necesidades y objetivos comunicativos de los y las estudiantes en la construcción del conocimiento. Los aportes del estudiantado son valorados e integrados en el curso.

Este curso se impartió de forma continua durante un año, entre enero y noviembre de 2016. En un principio, las actividades de voluntariado se efectuaban todas las semanas, a cada actividad se le dedicaban dos horas por semana. Luego de una revisión parcial del proyecto, se determinó que era importante dedicar tiempo al planeamiento de las actividades, para que los estudiantes se empoderaran y tomaran responsabilidad en el proceso de inmersión con la comunidad. También, se observó que una actividad por semana requería apoyo logístico adicional para el traslado de los estudiantes del campus al lugar donde se desarrollaría el evento y se percibió cansancio de los discentes debido a que algunos de estos transportes, causado por los problemas de tráfico en la ciudad de San José, tomaban hasta una hora.

Por esto, se determinó asignar cuatro de las ocho horas de actividades de voluntariado al planeamiento de la actividad. Esto permitió trabajar con los estudiantes de forma más explícita y estructurada su proceso de concienciación respecto de la situación y la población con la que interactuaban, definir de forma más precisa los objetivos lingüísticos de la actividad y elaborar de manera más completa las guías de trabajo con la comunidad.

En una versión final, las horas dedicadas el voluntariado se asignaron de la siguiente manera:
Tabla 1. Distribución de las horas de actividades de ApS

\begin{tabular}{|c|c|c|c|}
\hline Semana 1 & Semana 2 & Semana 3 & Semana 4 \\
\hline $\begin{array}{c}\text { Planeamiento } \\
\text { actividad 1 } \\
2 \text { horas }\end{array}$ & $\begin{array}{c}\text { Actividad 1 } \\
2 \text { horas }+ \\
\text { traslado }\end{array}$ & $\begin{array}{c}\text { - Retroalimentación } \\
\text { actividad 1. } \\
\text { - Planeamiento } \\
\text { actividad 2 horas }\end{array}$ & $\begin{array}{c}\text { Actividad 2 } \\
\text { Retroalimentación } \\
\text { 2 horas + traslado }\end{array}$ \\
\hline
\end{tabular}

Fuente: Elaboración propia.

En total, se efectuaron alrededor de 20 actividades de voluntariado durante el periodo enero-noviembre 2016. En cuanto a los tipos de actividades y las poblaciones con las que se trabajó en este lapso, estas son algunas de las que se efectuaron:

- Visitas a un hogar para adultos mayores: se realizaron actividades varias como bingos, celebraciones de eventos patrios, conversatorios, entre otros intercambios.

- Cooperación con ANPA (Asociación Nacional Protectora de Animales): los estudiantes participaban en las capacitaciones en escuelas públicas a niños y niñas en edad escolar sobre cómo ser dueño responsable de mascotas (perros y gatos). Esta iniciativa pretende disminuir la presencia de animales abandonados en las calles de la capital costarricense a partir de actividades educativas dirigidas a niños y niñas y encaminadas a prevenir y erradicar el maltrato animal.

- Cooperación con el Programa Integral del Adulto Mayor de la Universidad de Costa Rica: intercambios de tipo tandem, entre los adultos mayores y los estudiantes.

- Visitas a finca agroecológica: los estudiantes tuvieron la oportunidad de interactuar con agricultores orgánicos costarricenses, recorrer la finca y ser parte del proceso de siembra y cultivo de productos orgánicos.

Debido a que las actividades de ApS tenían como fin apoyar el mejoramiento de habilidades lingüísticas y contribuir en la sensibilización de los estudiantes respecto de comunidades o grupos en condiciones de vulnerabilidad o diferentes al grupo etario de los estudiantes, al finalizar los cursos de Conversación con proyección a la comunidad se aplicó un instrumento de evaluación a cada estudiante. Dicho instrumento buscaba evaluar la percepción de este en 
el mejoramiento de sus habilidades lingüísticas y en su proceso de sensibilización posterior a su participación en las actividades de ApS. Los resultados de estas encuestas, así como la metodología empleada en el análisis se discuten en el siguiente apartado.

\section{PERCEPCIÓN DE LAS ACTIVIDADES DE APS POR PARTE DE LOS ESTUDIANTES}

Como parte del proceso de evaluación regular del Programa de Español, a los estudiantes se les solicita llenar un formulario cuyo fin es conocer su opinión respecto del desempeño docente, la elaboración del programa, los recursos didácticos empleados, la atención del personal, entre otros, cuando terminan un curso. Para los cursos de Conversación con proyección a la comunidad I, II y III, a partir del 2016, a ese instrumento se le incluyeron tres preguntas. La primera, preguntaba al estudiante si había participado en las actividades de voluntariado; la segunda estaba relacionada con la percepción del estudiante respecto de su nivel de lengua antes y después de las actividades de voluntariado. En concreto: "En su opinión, ¿las actividades de voluntariado ayudaron a mejorar sus habilidades en Español? Por favor, explique.". La tercera pregunta buscaba obtener la percepción del discente respecto de su proceso de sensibilización con comunidades costarricenses en condición de vulnerabilidad o diferentes al grupo etario del estudiante. Específicamente: "En su opinión, ¿las actividades de voluntariado le ayudaron a sensibilizarse más con comunidades costarricenses más vulnerables? Por favor, explique.".

Es importante aclarar que, aunque en la evaluación se utilizó el término "voluntariado", las actividades efectuadas se enmarcan en un programa de ApS; la razón por la cual se utilizó es porque se consideró que los estudiantes estarían más familiarizados con el término "voluntariado" que con el ApS.

Un total de 66 estudiantes afirmaron haber participado de las actividades de ApS. De estos, el 75\% (50) cree que sí le ayudaron a mejorar sus habilidades en español, $18 \%$ (12) no lo creen y $6 \%$ (4) no contestaron (véase gráfico 1). En relación con la tercera pregunta, el $81 \%$ (54) de ellos considera que las actividades de ApS sí le ayudaron a sensibilizarse con las comunidades costarricenses más vulnera- bles, 9\% (6) dijo que no y 9\% (6) no contestó (véase el Gráfico 2).

\section{Gráfico 1: Mejora de las habilidades de español}

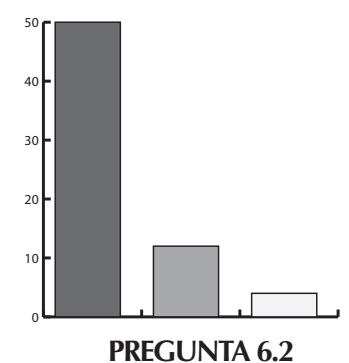

sí $\square$ No $\square$ SIN CONTESTAR

\section{Gráfico 2: Sensibilización de las comunidades costarricenses vulnerables}

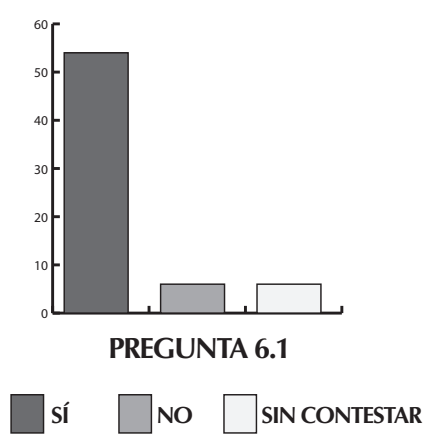

\section{ANÁLISIS DE LOS COMENTARIOS}

Para analizar los comentarios de ambas preguntas, se dividieron las opiniones en positivas y negativas. Para establecer la diferencia, en primer lugar, se tomó en cuenta el uso del adverbio de afirmación o de negación y seguidamente se determinó el tipo de comentario.

Además, para establecer estas categorías se tomaron como base las variables usadas en investigaciones anteriores y lo encontrado en una primera revisión de la muestra. Por ejemplo, para el análisis de los comentarios sobre la mejora en las habilidades lingüísticas se proponen: señala la importancia de interactuar con hablantes nativos, responde a la pregunta (no incluye información adicional), lo/ la motiva a hablar con otros hablantes nativos, lo/ la ayuda a comprender variedades del español y a enriquecer el vocabulario, permite interactuar más con compañeros de clase y profesor(a), dificultad 
para comunicarse con hablantes nativos, disconformidad con la organización, falta de tiempo.

En cambio, para el análisis de la percepción del proceso de sensibilización se definieron las siguientes categorías: relacionado con la práctica de español; es bueno, divertido o interesante, se relaciona con una emoción o sentimiento, se refiere su proceso de sensibilización; recalca la posibilidad que de otra forma no tendría; dificultad para comunicarse; no sintió diferencia; disconformidad con la organización; fue aburrido.

\section{PERCEPCIÓN DE LA MEJORA DE SUS HABILIDADES EN ESPAÑOL}

Se encontró un total de 20 comentarios relacionados con la mejora de las habilidades lingüísticas y un total de 25 comentarios que se referían al proceso de sensibilización. En el primer grupo, 16 fueron clasificados como positivos y siete como negativos. Algunos comentarios estaban conformados por un enunciado positivo y otro negativo, en estos se contabilizaron de forma independiente, por esto, la suma de los datos por separado podría ser mayor al número total de comentarios.

En cuanto a los positivos, la mayoría de los encuestados (diez) señaló la importancia de interactuar con hablantes nativos como la razón principal para participar en este tipo de actividades y como la causa de la mejoría que percibieron en sus habilidades lingüísticas. Por ejemplo:

- Sí, porque hablar con nativos es importante.

- Yes, the activities gave me the opportunity to speak to locals.

- Sí, porque estaba hablando con los participantes y fue buena práctica.

Estos comentarios evidenciaron también que los estudiantes se sintieron forzados a hablar la lengua durante las actividades, lo cual es calificado como positivo:

- They helped in the way that we are forced to make conversations with native speakers, which is very important.

- Yes, because you are forced to speak Spanish, otherwise they don't understand you.
- Yes it did. It's always helpful when you are forced to speak Spanish.

Las actividades de ApS, como se observa en los comentarios -siguiendo a Tacelosky (2013) - impulsaron a los estudiantes a cruzar las barreras lingüísticas conforme utilizaban la lengua meta en un contexto real. Esto podría llegar a incidir en su motivación por seguir estudiando o practicando la lengua:

Research has shown that using the target language in a rich and meaningful way with native speakers in a service setting can increase student motivation for language study (Barreneche y Ramos-Flores, 2013).

En el resto de comentarios (seis) se encontraron razones varias para justificar la percepción de la mejoría en las habilidades lingüísticas:

- Algunas personas se limitaron a responder afirmativamente a la pregunta a partir de respuestas completas: Sí, mejora sus habilidades en español.

- Otros afirmaron haber comenzado a experimentar con el español desde que se integraron en las actividades de ApS: Sí, he experimentado mucho últimamente.

- En algunos casos, afirmaron que la experiencia de ApS les había ayudado a comprender variedades del español y a enriquecer su vocabulario: Yes, because it helps understand the local dialect / Yes, I think so because there was a different type of vocabulary I had to use in class so it challenged me more.

- Una persona rescató la posibilidad de interactuar más con su profesor(a) y compañeros de clase: Yes, provided extra time to converse with friends and professors.

Los comentarios categorizados como negativos se relacionaron, principalmente (cuatro de seis) con la dificultad que les representó comunicarse con hablantes nativos:

- No, es difícil para hablar español.

- Casi no conversamos.

Una persona se quejó de la organización de las actividades. Puig (2015) ya se había referido en su trabajo a este aspecto. Según este autor, los proyectos 
de ApS deben estar abiertos a cambios imprevistos. El trabajo con organizaciones sociales requiere de mucha organización previa; sin embargo, podrían ocurrir situaciones inesperadas que compliquen su ejecución. Por ejemplo, en dos ocasiones, los proyectos con escuelas públicas en coordinación con ANPA para la educación de niños y niñas en tenencia responsable de mascotas fueron cancelados a última hora. En otras ocasiones, la institución anfitriona no estuvo preparada para recibir al grupo. Para los estudiantes de los programas de español, quienes en su mayoría vienen de los Estados Unidos y Europa, estos imprevistos son difíciles de comprender.

\section{PERCEPCIÓN DE SU PROCESO DE SENSIBILIZACIÓN CON COMUNIDADES EN CONDICIÓN DE VULNERABILIDAD}

En este grupo, 21 comentarios fueron clasificados como positivos y cinco como negativos. Se repitió la tendencia a que algunos comentarios estuvieran conformados por un enunciado positivo y otro negativo, en cuyo caso, se procedió a contabilizarlos de forma independiente. En cuanto a las respuestas, a pesar de que en esta pregunta se consultó a los encuestados específicamente por su proceso de sensibilización, algunos de ellos (cuatro) se refirieron nuevamente al aspecto lingüístico:

- It was useful to talk to native speakers.

- Yes, because it involves practice of conversation.

Sin embargo, la mayoría (ocho) hizo referencia a su proceso de sensibilización:

1. Had a great learning about the vulnerable population of the Costa Rican communities.

2. Podía ver los problemas.

3. It was a beneficial experience to see how environmentally conscious Costa Rican's are making efforts to improve and maintain Costa Rica's natural environment.

4. Actually seeing them and talking to them is always better than just talking about them.

5. The activities helped me to understand the community better.
6. It gave me a good insight into other parts of the country and culture.

7. Las actividades me dieron el conocimiento cómo funciona la sociedad de CR.

8. Yes, because I can see some important places of Costa Rica.

De estos comentarios, se rescata, en primer lugar, la visualización, por parte de los discentes, de situaciones o poblaciones de la sociedad costarricense que antes no conocían (comentarios 2, 3, 6 y 8), así como un proceso reflexivo sobre la necesidad de la acción fuera del aula como instrumento de cambio social (comentario 4) y una idea de que entrar en contacto con las comunidades permite ampliar el conocimiento cultural y entender las diferencias (comentarios 1, 5 y 7). Se observan en los datos, actitudes que podrían indicar el inicio o el fortalecimiento de un proceso de concienciación. Este, como vimos líneas más arriba, comienza cuando el individuo se apodera de la realidad (Freire, 2015) y para esto debe entrar en contacto con ella con todos sus sentidos.

La educación, entonces, debe trascender las discusiones sobre las desigualdades y las injusticias y comenzar a actuar en ellas desde la agentividad social; comentarios como el registrado en la línea cuatro muestran que la institución educativa puede y debe impulsar prácticas que permitan a los estudiantes comprometerse con su transformación y con la de su entorno en uno mejor y más equilibrado.

Respecto de los comentarios 1, 5 y 7, Barreneche y Ramos (2013) ya habían analizado en su estudio sobre la relación entre el ApS y el incremento en la comprensión de aspectos culturales, para ellos: "service-learning projects in local Hispanic communities provide opportunities for students to engage with native speakers, reflect on and understand cultural differences" (p. 220). Un mejor entendimiento de las culturales incidirá directamente en una mejor habilidad para comunicarse (Freire, 2012).

De los ocho comentarios restantes, tres relacionaron el proceso de sensibilización con lo ventajoso de tomar ese curso, sin el cual no habrían tenido la oportunidad de visitar estas comunidades:

- Helped to go somewhere we would not have gone otherwise. 
- Me gusta hablar con ticos, cuales normalmente no encontraría.

- Para mí, fueron buenas experiencias porque no podía participarles si no soy estudiante de la UCR.

Al respecto, Tacelosky (2013) menciona que los proyectos basados en la comunidad les dan la oportunidad a los estudiantes de aplicar lo que están aprendiendo en la clase, de forma que hay mejoras en su propio aprendizaje en maneras que de otra forma no se alcanzarían. Es decir, el ApS brinda conocimientos que se integran en la experiencia de vida, precisamente a partir de estas actividades.

Los otros comentarios en este punto calificaban la experiencia como buena, divertida o interesante o se referían a la percepción desde una emoción ("me encantó" / "fue una experiencia maravillosa", por ejemplo). En cuanto a los comentarios negativos (cinco en total), surgieron elementos en común con la pregunta dos, en concreto, la dificultad para comunicarse y las quejas respecto de la organización. Además, una persona no sintió diferencia en su sensibilización antes y después de las actividades y otra persona calificó el ApS como "aburrido".

\section{CONCLUSIONES}

El Aprendizaje Servicio (ApS) es una metodología de enseñanza que busca integrar en el proceso de aprendizaje experiencias con la comunidad con el objetivo de que los estudiantes practiquen o mejoren su desempeño en la lengua y que desarrollen o mejoren su conciencia con respecto a las situaciones de desigualdad o injusticia en la sociedad. Se basa en una perspectiva de la educación en la que esta es un proceso que debe contribuir en la formación de seres humanos que busquen transformarse y transformar su entorno a partir de la noción de agentividad social para el cambio.

Los resultados de las experiencias de ApS en Europa, Estados Unidos e Hispanoamérica mostraron el establecimiento de una relación entre los programas de ApS y el cumplimiento de los objetivos académicos, así como actitudes positivas hacia la lengua meta, sus hablantes; un involucramiento mayor en el proceso de aprendizaje y en la comunidad.
La investigación descrita líneas arriba presenta los resultados de la aplicación de un proyecto de ApS en el Programa de Español como Lengua Extranjera de la Universidad de Costa Rica por medio del curso "Conversación con proyección a la comunidad I, II y III". Se midió la percepción de los estudiantes de acuerdo con dos variables, la mejora en sus habilidades lingüísticas y su proceso de sensibilización con comunidades en condición de vulnerabilidad.

Las encuestas muestran una valoración positiva del tiempo invertido en actividades de ApS. Los comentarios positivos en la parte académica resaltan, en mayor grado, la importancia de interactuar con hablantes nativos en el mejoramiento de las competencias en la lengua meta y, con menor frecuencia, se encontraron comentarios que apelaban a un deseo de experimentar más con la lengua, a las ventajas de contar con más tiempo para compartir con los otros compañeros y la profesora del curso y a la exposición a otras variedades del español.

En relación con el proceso de sensibilización, los resultados mostraron que, en general, las actividades de ApS contribuyeron a crear un espacio que, de acuerdo con Escofet, Folgueiras, Luna y Palou (2016), promueve responsabilidades entre los distintos participantes en el proceso educativo que desembocan en una formación integral, hacia el desarrollo de competencias sociales y éticas, basada en aprendizaje significativo. Los estudiantes lograron, en su mayoría, enlazar el mejoramiento de sus habilidades lingüísticas con su proceso de transformación en agentes sociales de cambio; en algunos casos, esto se consiguió desde la concienciación (Freire, 2015), es decir, el estudiante no tenía conocimiento de la existencia de la comunidad o de la situación y, a partir de las actividades de ApS, lo adquirió. Otros reflexionaron sobre la necesidad de recurrir a la acción fuera del aula para trascender la discusión sobre las comunidades y transformarla en actuación y otros afirmaron haber ampliado su comprensión de las diferencias culturales.

Los comentarios negativos estuvieron relacionados, en su mayoría, con la dificultad para expresarse en la lengua meta o con la disconformidad con aspectos de la organización de las actividades.

El proyecto presentado de ApS evidenció que la educación requiere salir de las aulas y transformarse 
en praxis si desea cumplir con los objetivos que la sociedad actual requiere: seres humanos conscientes, libres, dispuestos a convertirse en agentes de cambio, comprometidos con su bienestar y el de la comunidad en la que se encuentran. El que los estudiantes de español como segunda lengua no estén en su país de origen no implica que deban permanecer ajenos a las problemáticas sociales, por el contrario, es una excelente oportunidad para el desarrollo de la empatía y la solidaridad, para la apertura hacia nuevas culturas. En resumen, una oportunidad de transformación.

El docente, como intelectual transformador, tiene la posibilidad -y la obligación- de comprometerse con su propio aprendizaje, el de sus estudiantes y con la sociedad en procura de convertir la experiencia pedagógica en un proceso que busque no solo logros a nivel académico sino transformación personal a través de la exposición a situaciones, textos y personas que permitan sensibilizar a los estudiantes y los impulsen a ser agentes de cambio. Desde una institución de educación superior, esta responsabilidad es aún mayor.

\section{RETOS Y RECOMENDACIONES}

Del trabajo realizado, se desprenden los siguientes retos y recomendaciones:

- Desarrollar un proceso de evaluación formativa en cada etapa del proyecto y dar seguimiento a todos los participantes: el grupo comunitario, el/la docente y los estudiantes; con esto, se pretende medir el impacto también en la comunidad y en los docentes. Hasta ahora, los estudios se concentran principalmente en los beneficios para los estudiantes, se menciona la comunidad, pero no se mide el impacto de la ApS a largo plazo. Se recomienda una evaluación exante, intermedia y ex-post.

- Las barreras administrativas son un reto en la ejecución de proyectos de ApS en instituciones públicas, el modelo educativo actual es poco receptivo en relación con iniciativas que involucran sacrificar horas lectivas de aprendizaje formal por horas, que en el ApS se conocen como de acción o de experiencia. El justificar estas actividades y conseguir las autorizaciones para llevarlas a cabo es un proceso largo, muchas veces tedioso y empapado de trámites burocráticos, al menos en Costa Rica.

- El trabajo con las organizaciones comunales es también un reto, como ya se comentó, algunas veces las actividades y los proyectos son cancelados, aun cuando han existido programación y planeamiento previos importantes. En otras ocasiones, las instituciones anfitrionas olvidan prepararse para la llegada de los estudiantes y esto genera sensación de desorganización. Se recomienda trabajar con los estudiantes este aspecto para familiarizarlos con la idea de que estos imprevistos podrían surgir y que son parte del aprendizaje y de la cultura.

- Para futuras investigaciones, se recomienda ampliar las variables de medición de la percepción: incluir la motivación para seguir estudiando español, uso de la lengua fuera de las actividades de ApS, inserción en actividades de este tipo posterior a su participación en el programa, entre otras.

- Ampliar los periodos de desarrollo de los proyectos de ApS podría ser una manera de medir el impacto a largo plazo; sin embargo, esto implicaría estadías más prolongadas de los estudiantes y en un contexto como el de enseñanza de lenguas en Costa Rica esto no es muy viable.

\section{REFERENCIAS BIBLIOGRÁFICAS}

Agray Vargas, N. (2010). La construcción del currículo desde perspectivas críticas: una producción cultural. Signo y pensamiento. México: Siglo XXIX Ediciones, 420-427.

Barreneche, G. I. y Ramos-Flores, H. (2013). Integrated or Isolated Experiences? Considering the Role of Service Learning in the Spanish Language Curriculum. Hispania. 96 (2), 215-228.

Bringle, R. y Hatcher, J. (1996). Implementing Service Learning in Higher Education. Journal of Higher Education. 67 (2), 221-239.

Dewey, J. (1977). Mi credo pedagógico. Buenos Aires: Centro Editor de América Latina.

Ebacher, C. (2013). Taking Spanish into the Community: A Novice's Guide to Service-Learning. Hispania, 96 (2), 397-408. 
Escofet, A.; Folgueiras, P.; Luna, E. y Palou, B. (2016). Elaboración y validación de un cuestionario para la valoración de proyectos de aprendizaje-servicio. Revista Mexicana de Investigación Educativa, 21 (70), 929-949.

Faszer-McMahon, D. (2013). Social networking, Microlending, and Translation in the Spanish Service-Learning Classroom. Hispania, 96 (2), 252263.

Freire, P. (2012). Pedagogía de la autonomía: saberes necesarios para la práctica educativa. México: Siglo Veintiuno.

Freire, P. (2015). Pedagogía Liberadora. Antología. Madrid: Catarata.

Giroux, H. (1988). Teachers as intelectuals: towards a critical pedagogy of learning. Connecticut: Bergin \& Gavery Publishers, Inc.

Grundy, S. (1987). Producto o praxis del curriculum. Madrid: Ediciones Morata.

Habermas, J. (1974). Teoría y praxix. Estudios de filosofía social. Madrid: Tecnos.

Hellebrant, J. y Jorge, E. (2013). The Scholarship of Community Engagement: Advancing Partnerships in Spanish and Portuguese. Hispania. 96 (2), 203214.

Jovanovic, A.y Filipovic, J. (2013). Spanish teacher Education Programs and Community Engagement. Hispania, 96 (2), 283-294.

Kincheloe, J. L. (1993). Toward a critical politics of teacher thinking. Westport: Bergin \& Garvey.

Kumaravadivelu, B. (2003). Beyond Methods. Macroestrategies for Language Teaching. London: Yale University Press.

Llidicoat, A. (2011). Language teaching and learning from an intercultural perspective. En: Hinkel, E. (Ed.). Handbook of research in second language teaching and learning, vol. II, 837-855. New York: Routledge.

Llorente, L. (2003). "Noticias locales", programa de servicios a la comunidad. Actas del XIV Congreso de la Asociación para la enseñanza del español como segunda lengua. Instituto Virtual Cervantes. España.
Mooney, L. A. y Edwards, B. (2001). Experiential Learning in Sociology: Service Learning and Other Community-Based Learning Initiatives. Teaching Sociology. 29 (2), 181-194.

Naval, C., García, R, Puig, J. y Santos, M. (2011). La formación ético-cívica y el compromiso social de los estudiantes universitarios. Encounters on Education. 12, 77-91.

Palincsar, Sullivan A. (1998). Social constructivism perspectives on teaching and learning. Annual Review of Psicology. 49, 345-375. DOI: 10.1146/ annurev.psych.49.1.345.

Pellettieri, J. (2011). Measuring language-related Outcomes of Community-based Learning in Intermediate Spanish Courses. Hispania, 94 (2), 285-302.

Puig, J. M. (2015). Cómo realizar un proyecto de aprendizaje servicio? 11 ideas clave. Barcelona: Graó.

Punset, E. (2011). ¿Por qué los niños se aburren en la escuela? (web) En: https://www.youtube.com/ watch?v=eEqF_1aXUw4.

Rodríguez, M. R. (2014). El aprendizaje-Servicio como estrategia metodológica en la Universidad. Revista Complutense de Educación, 25 (1), 5-113.

Rosas, R. (2008). Piaget, Vigotsky y Maturana: constructivismo a tres voces. Buenos Aires: Aique Grupo Editor.

Sotelino Lozada, A. (2014). Aprendizaje-servicio en las universidades gallegas. Evaluación y propuesta de desarrollo (Tesis doctoral). Universidad de Santiago de Compostela.

Tacelosky, K. (2013). Community-based ServiceLearning as a Way to Meet the Linguistic Needs of Transnational Students in Mexico. Hispania, 96 (2), 328-341.

Zapata, G. (2011). The Effects of Community Service Learning Projects on L2 Learners' Cultural Understanding. Hispania, 94 (1), 86-102. 